Check for updates

1 Blackburn with Darwen Borough Council

Cite this as: $B M J 2022 ; 376: 0100$ http://dx.doi.org/10.1136/bmj.0100 Published: 14 January 2022

\title{
What is driving all cause excess mortality?
}

\section{Omicron is likely to generate more avoidable deaths from non-covid causes than from covid, a risk that has not been communicated clearly, writes Dominic Harrison}

\section{Dominic Harrison professor and director of public health and wellbeing}

The first purpose of using non pharmaceutical interventions to control the spread of an infectious disease is to ensure that the health and care system is not overwhelmed. This is critical to avoiding loss of capacity to deliver urgent life-saving treatment for all causes of avoidable mortality.

With the current surge of the omicron variant of SARS-CoV-2, we have again acted with too little control, too late. That large parts of the health and care system will be overwhelmed is now inevitable, and in this wave particularly, covid deaths may only be a lesser part of the subsequent avoidable mortality.

The levels of the population who risk exposure to coronavirus are a political choice. Despite all receiving the same advice and data from the UK Health Security Agency in late autumn 2021, England, Scotland, Wales, and Northern Ireland have made different choices about how to manage the impact of the omicron variant. Fewer controls implemented later than required have generated avoidably higher case rates in England. The ONS UK Coronavirus (COVID-19) Infection Survey for the 5 January 2022 shows that for the week ending 31 December 2021, England had 1 in 15 people infected Wales and Scotland 1 in 20; Northern Ireland 1 in $25 .^{1}$

The increased transmissibility of omicron compared to earlier variants has generated a more rapid surge in cases than previous waves placing both hospital and out of hospital systems in a double jeopardy of simultaneous high demand and reduced capacity due to staff shortages. Critical incidents are an imperfect indicator of acute hospitals' operating status as tight management controls by NHS England may restrict "local declarations"; but as of the 6 January, 24 out of 137 NHS acute trusts had already declared a critical incident. It is inevitable that more critical incidents will emerge and the UK prime minister, Boris Johnson, has himself advised that "parts of the NHS may be overwhelmed."2

Omicron may cause fewer hospital admissions as a percentage of all cases than previous variants, largely due to higher levels of population immunity, but much of that benefit has been squandered through allowing a higher, and more rapid, rise in case rates. The current overwhelming of the health and care system, and the further disruption to come, as cases and staff absences rise potentially into late January and early February 2022, is now likely to generate more avoidable deaths from non-covid causes than from covid. The risk and reality of this effect is not being clearly communicated in the public domain as part of the UK's "pandemic related mortality" reporting.
In my own Borough of Blackburn with Darwen in the week ending 1oth December-just at the early stage of the Omicron surge-there was just one covid death and six excess non-covid deaths from "all-causes" when compared to the average of the previous five years. The national Covid Actuaries Group reported at the end of 2021 that "Latest ONS deaths data (to week ending 17 December) showed that for England and Wales, 1,650 more deaths were recorded in-week compared to the 2015-19 year average. That's $15 \%$ higher." 3 The number of non covid deaths arising from "excess mortality" will undoubtedly rise dramatically over the next three months as a result of the health and care system being overwhelmed.

We have seen this scenario before and ignored its lessons. One of the largest spikes in "all cause excess mortality" since the Second World War was in January 2015. Research on the likely causes of that event generally found that high staff vacancies in the NHS, high staff absences, a lack of beds, inadequate out of hospital care capacity in social care and a simultaneous surge in demand generated catastrophic declines in all the metrics of safe effective care. ${ }^{4}$ In the winter of 2015, but most notably January 2015, performance plummeted to the point that those who were in immediate need of urgent and emergency care received significantly delayed or sub-optimal access to life saving treatment. The subsequent very high excess mortality was a consequence of the inability of the health and care system to match the surge in demand with an appropriate surge response. The report also highlighted the exacerbation to this risk from an increased vulnerability in the population arising from welfare benefit cuts and sustained underfunding of adult social care.

All of the identified risk condition metrics associated with a surge in all cause excess mortality in January 2015 have been flashing red since the autumn of 2021. Despite two years of pandemic impacts and three variants of concern of SARS-CoV-2 in the last 12 months, we have yet to see any strategic plan emerge defining what we might need to do to "live with the virus." Whatever that is going to look like, it cannot look like the current situation.

When, and if, such a national strategy emerges, we will also need it to address how the health and care system, both in and out of hospital, can be designed and resourced in such a way as to be capable of managing surge demand with surge capacity.

This will be critical to saving lives and livelihoods in the years to come. 


\section{OPINION}

Provenance and peer review: commissioned, not peer reviewed

1 Office for National Statistics. Coronavirus (COVID-19) Infection Survey headline results, UK https://www.ons.gov.uk/peoplepopulationandcommunity/healthandsocialcare/conditionsanddiseases/bulletins/coronaviruscovid19infectionsurveypilot/latest

2 The Guardian. Parts of NHS may be overwhelmed by Covid wave, admits Boris Johnson. Jan 2022. https://www.theguardian.com/world/2022/jan/04/parts-of-nhs-may-be-overwhelmed-bycovid-wave-admits-boris-johnson

3 Twitter. Stuart Mcdonald on Twitter: "Latest ONS deaths data (to week ending 17 December) has been released. 1,650 more deaths were recorded in-week compared to the 2015-19 year average. That's $15 \%$ higher. Year-to-date there have been 564,409 deaths recorded, which is 10\% higher than the 2015-19 average. https://t.co/Fmit9kXi2

4 Hiam L, Dorling D, Harrison D, McKee M. What caused the spike in mortality in England and Wales in January 2015?J R Soc Med 2017;110:131-7

doi: 10.1177/0141076817693600. pmid: 28208024 